\title{
Eclipses of Jupiter's Satellites
}

observed with the $4^{1 / 2}$ inch Equatorial of the Windsor Observatory, N. S. Wales.

\begin{tabular}{|c|c|c|c|c|c|}
\hline \multirow{2}{*}{$\frac{1885}{\text { Feb. } 25}$} & \multicolumn{2}{|c|}{ Sat. } & \multirow{2}{*}{$\frac{\text { Pow. }}{94}$} & \multirow{2}{*}{$\frac{\text { Winds. M. T. }}{8^{\mathrm{h}} 54^{\mathrm{m}} 4^{2^{\mathrm{s}}} \cdot 4}$} & \multirow{2}{*}{$\frac{\text { Corr. N. A. }}{-0^{\mathrm{m}} \quad 4 \cdot 4}$} \\
\hline & II & $\mathrm{R}$ & & & \\
\hline lar. Io & III & $\mathbf{R}$ & 190 & $\begin{array}{lll}8 & 53 & 48.5\end{array}$ & +335.7 \\
\hline 18 & I & $\mathrm{R}$ & 190 & $\begin{array}{lll}8 & 43 & 53.2\end{array}$ & +0 \\
\hline 25 & I & $\mathbf{R}$ & 190 & 103825.2 & to \\
\hline 29 & II & $\mathbf{R}$ & 190 & 839 & to 20.9 \\
\hline ril 5 & II & $\mathbf{R}$ & 190 & $\begin{array}{lll}11 & 142.8\end{array}$ & -0 \\
\hline 22 & III & $\mathrm{R}$ & 190 & $\begin{array}{lll}8 & 44 & 13.8\end{array}$ & +137.0 \\
\hline 23 & II & $\mathbf{R}$ & 190 & $\begin{array}{lll}5 & 44 & 27.9\end{array}$ & $--0 \quad 18.9$ \\
\hline
\end{tabular}

\begin{tabular}{|c|c|c|c|c|c|}
\hline \multirow{2}{*}{$\frac{1885}{\text { April } 26}$} & \multicolumn{2}{|c|}{ Sat. } & \multirow{2}{*}{$\frac{\text { Pow. }}{190}$} & \multirow{2}{*}{$\frac{\text { Winds. M. T. }}{7^{\mathrm{h}} 14^{\mathrm{m}} 4^{8} 8^{5} .9}$} & \multirow{2}{*}{$\frac{\text { Corr. N.A. }}{-0^{m} \quad 0^{s} .9}$} \\
\hline & I & $\mathrm{R}$ & & & \\
\hline 30 & II & $\mathrm{R}$ & 190 & $820 \quad 15.2$ & -0 22.6 \\
\hline May & I & $\mathrm{R}$ & 190 & 9 IO 22.1 & to 32.3 \\
\hline 19 & I & $\mathrm{R}$ & 180 & $\begin{array}{lll}7 & 28 & 56.6\end{array}$ & +0 1 2.8 \\
\hline 25 & II & $\mathrm{R}$ & 180 & 52431.1 & $-0 \quad 29.7$ \\
\hline 26 & I & $\mathrm{R}$ & 180 & $\begin{array}{lll}9 & 24 & 4.4\end{array}$ & to 12.6 \\
\hline $3^{1}$ & IV & $\mathbf{R}$ & 180 & $63^{8} \times 4.5$ & +151.7 \\
\hline
\end{tabular}

Remarks.

Feb. 25. Clear: satellite close to planet's limb, and well defined.

Mar. ro. Planet well defined, but faint owing to thin cloud; belts barely distinguishable when satellite first detected.

18. Beautifully clear, with images steady and well defined: observation probably a second late.

25. Beautifully clear, with images fairly steady and defined: moon near first quarter.

29. Fair definition: reappearance seen through thin cloud, which did not obscure the planet's belts.

April 5. Clear, but images rather tremulous.

22. Definition and steadiness excellent: recorded time rather uncertain, but satellite certainly seen seven seconds later.

23. Beautifully clear, but twilight strong and moon near planet; planet boiling, but belts plainly seen.

26. Beautifully clear but moon very bright: steadiness and definition excellent.

Observatory, Windsor, N. S. Wales ${ }_{1} 885$ Sept. I 4.
April 30. Cloudless, with full moon: steadiness and definition excellent: noted time probably a second late.

May 3. Sky apparently clear, but planet slightly dimmed owing probably to haze.

19. Definition good and images steady.

25. Beautifully clear: steadiness and definition excellent, but twilight strong and moon present. Noted time doubtful, but certain of the satellite nine seconds later.

26. Beautifully clear: definition good and images steady, but moon very bright.

3r. Very slightly hazy, but steadiness and definition unusually good. The satellite attained full brightness about $6^{\mathrm{h}} 44^{3} /^{\mathrm{m}}$ but afterwards varied in lustre as compared with the other satellites. After fading again it attained a second maximum about $6^{\mathrm{h}} 4^{83} / 4^{\mathrm{m}}$.

The longitude adopted for comparison of the observations with the Nautical Almanac is $10^{\mathrm{h}} 3^{\mathrm{m}} 20^{3} .8$ as given in Dr. Auwers' paper in Nr. 2635-6 of the Astr. Nachr.

\section{Bemerkung zu dem Schreiben von Dr. J. L. E. Dreyer (A. N. 2704), betreffend den neuen Stern im grossen Andromeda-Nebel.}

Da die sehr dankenswerthe Mittheilung von Dr. Dreyer in Nr. 2704 der A. N. leicht die Auffassung veranlassen kann, als ob ich mich des in derselben berichtigten Irrthums schuldig gemacht hätte, so möchte es mir gestattet sein, auf meine Mittheilung in Nr. 2690 zurückverweisen zu dürfen, in welcher ich ausdrücklich mein Bedauern ausgesprochen habe, dass ich das Original der d'Arrest'schen Beschreibung nicht einsehen konnte. Wenn Dr. Dreyer meine Wahrnehmung vom 20. August für eine zweifellose Bamberg 1886 Febr. I 3 .
Folge einer optischen Täuschung hält, so muss ich dagegen bemerken, dass ich diese Wahrnehmung für eine zweifellose Grundlage meiner Ueberzeugung und Behauptung von der gleichzeitig mit dem Aufleuchten der Nova erfolgten Veränderung der sogenannten Nebelmitte halte, da diese Beobachtung, wie die späteren, mit aller Vorsicht und Sorgfalt an einem vorzüglichen Fernrohr, wie ts der Dorpater Refractor ist, gemacht wurde.

\section{Fohn Tebbutt.}

